\title{
ON THE CYLINDRICITY MEASUREMENT BY THE V-BLOCK METHOD
}

\author{
STEPIEN, K.; JANECKI, D. \& ADAMCZAK, S.
}

Abstract: In various branches of industry (for example, in paper, electric or ship industry), large and heavy cylindrical elements play very important role. Sometimes, they may be deformed under certain operating conditions, e.g. due to a high temperature or great external or internal loads. Consequently, wear and damage of the working surfaces are observed. Therefore cylindricity deviations of such elements should be measured during their manufacturing and work. However, some cylinders cannot be measured accurately by existing measuring instruments because they are too large or too heavy and therefore they cannot be placed on the measuring table of the instrument. This is why the industries manufacturing or applying cylinders expect that measurements of cylindricity profiles will be made directly on the machine tool or in the work area.

Similar problem concerning roundness measurements was successfully solved by applying so-called V-block method. Therefore authors assumed that problem of in-situ cylindricity measurements can be also solved this way and they developed the concept of cylindricity measurements by the V-block method. The paper presents developed concept of V-block cylindricity measurements, results of simulations, experiments and conclusions.

Key words: cylindricity, measurement, V-block, method, deviation
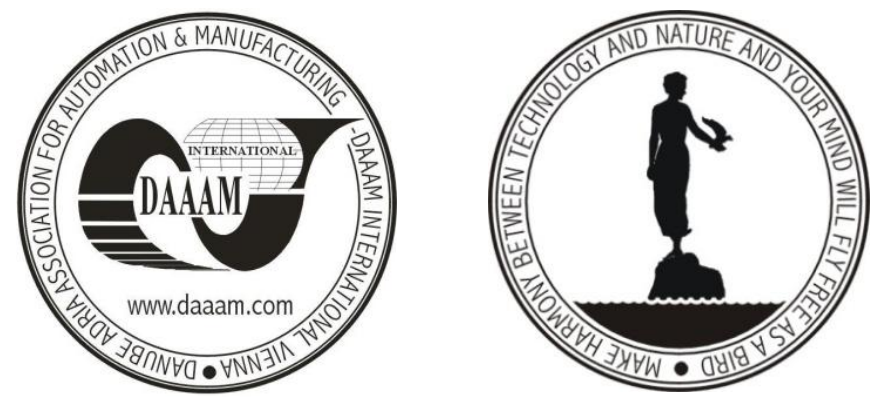

Authors' data: Dipl.-Ing. Dr. techn. Stepien, K[Krzysztof]*; Univ. Prof. Dipl.-Ing. Dr. techn. Janecki, D[ariusz]*; Univ.Prof. Dipl.-Ing. Dr.h.c.mult. Dr.techn. Adamczak, S[tanislaw]*, *Kielce University of Technology, Al. 1000-lecia P. P. 7, 25-314 Kielce, Poland,kstepien@tu.kielce.pl,djanecki@tu.kielce.pl, adamczak@tu.kielce.pl

This Publication has to be referred as: Stepien, K[rzysztof] \& Janecki, D[ariusz] \& Adamczak, S[tanislaw] (2012). On the Cylindricity Measurement by the V-block Method, Chapter 03 in DAAAM International Scientific Book 2012, pp. 027-044, B. Katalinic (Ed.), Published by DAAAM International, ISBN 978-3-901509-86-5, ISSN 1726-9687, Vienna, Austria

DOI: $10.2507 /$ daaam.scibook.2012.03 
Stepien, K.; Janecki, D. \& Adamczak, S.: On the Cylindricity Measurement by the ...

\section{Introduction}

The knowledge of the measurement of cylindricity profiles is relatively limited. The works available on the subject mainly concern the application of radial methods. The radial methods can be divided into two main groups: ones employing a rotary sensor, and ones employing a rotary table (see Fig. 1).
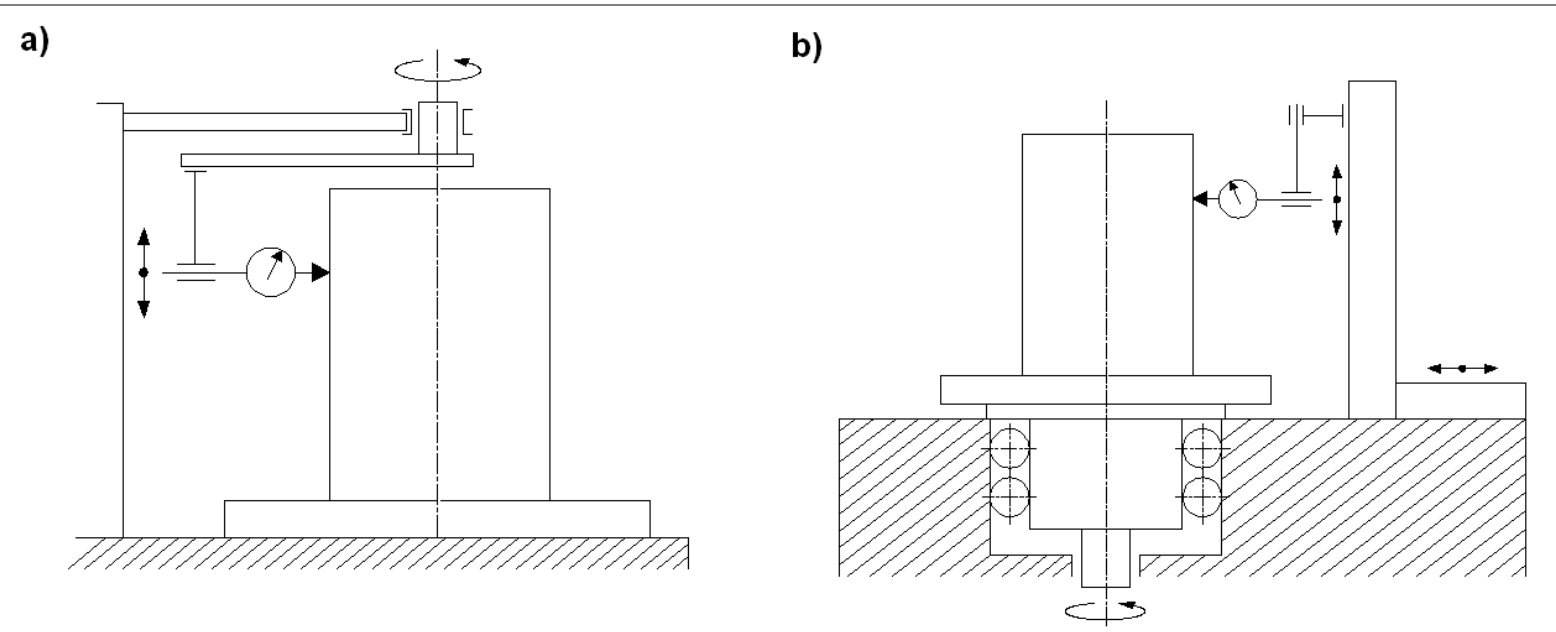

Fig. 1. Radial cylindricity measurements: a) using the rotary sensor; b) using the rotary table

The radial measurements of cylindricity profiles are of a high metrological level. Characterised by high accuracy, they provide complete information of the analysed surface (Zhao et al., 2006). However, the radial measuring instruments are applied only if it is possible to place the measured element on the measuring table.

In various branches of industry (for example, in paper, electric or ship industry), large and heavy cylindrical elements play very important role (Runje \& Medic, 2011). Such parts should posses very good quality of form and dimensions. Sometimes, they may be deformed under certain operating conditions, e.g. due to a high temperature or great external or internal loads. Consequently, wear and damage of the working surfaces are observed. Therefore cylindricity deviations of such cylinders should be measured during their manufacturing and work (Beranek et al., 2011). However, some of the elements cannot be measured accurately by existing measuring instruments because they are too large or too heavy and therefore they cannot be placed on the measuring table of the instrument. This is why the industries manufacturing or applying cylinders expect that measurements of cylindricity profiles will be made directly on the machine tool or in the work area (Gao \& Kiyono, 1997; Muralikrishnan, 2005). Problem of in-situ roundness measurements was successfully solved by applying so-called V-block method (Okuyama et al., 2003). Therefore authors assumed that also problem of in-situ cylindricity measurements can be solved this way. This is why the concept of cylindricity measurements by the V-block method has been developed.

In V-block methods a measuring signal is measured in relation to a physical datum. The datum is constituted by contact points of the V-blocks and the measuring sensor 
with the cylinder. Mutual location of these points in relation to the assigned coordinate system is defined by method parameters - angles $\alpha$ and $\beta$ (see Fig. 2). Traditional V-block methods used for measurement of roundness deviation are not accurate; they are suitable for rough evaluation only. However, application of FFT algorithm can eliminate main shortcomings of methods of this type.

In hitherto practice the V-block was used usually to measure simplified cylindricity. The aim of the authors of this paper was development of the V-block method that would allow evaluation of the entire surface of the element (the number of the cross-sections would be defined by the user).

\section{Concept of the cylindricity measurement by the V-block method}

The developed concept of cylindricity measurements by the V-block method is shown in Fig. 2.

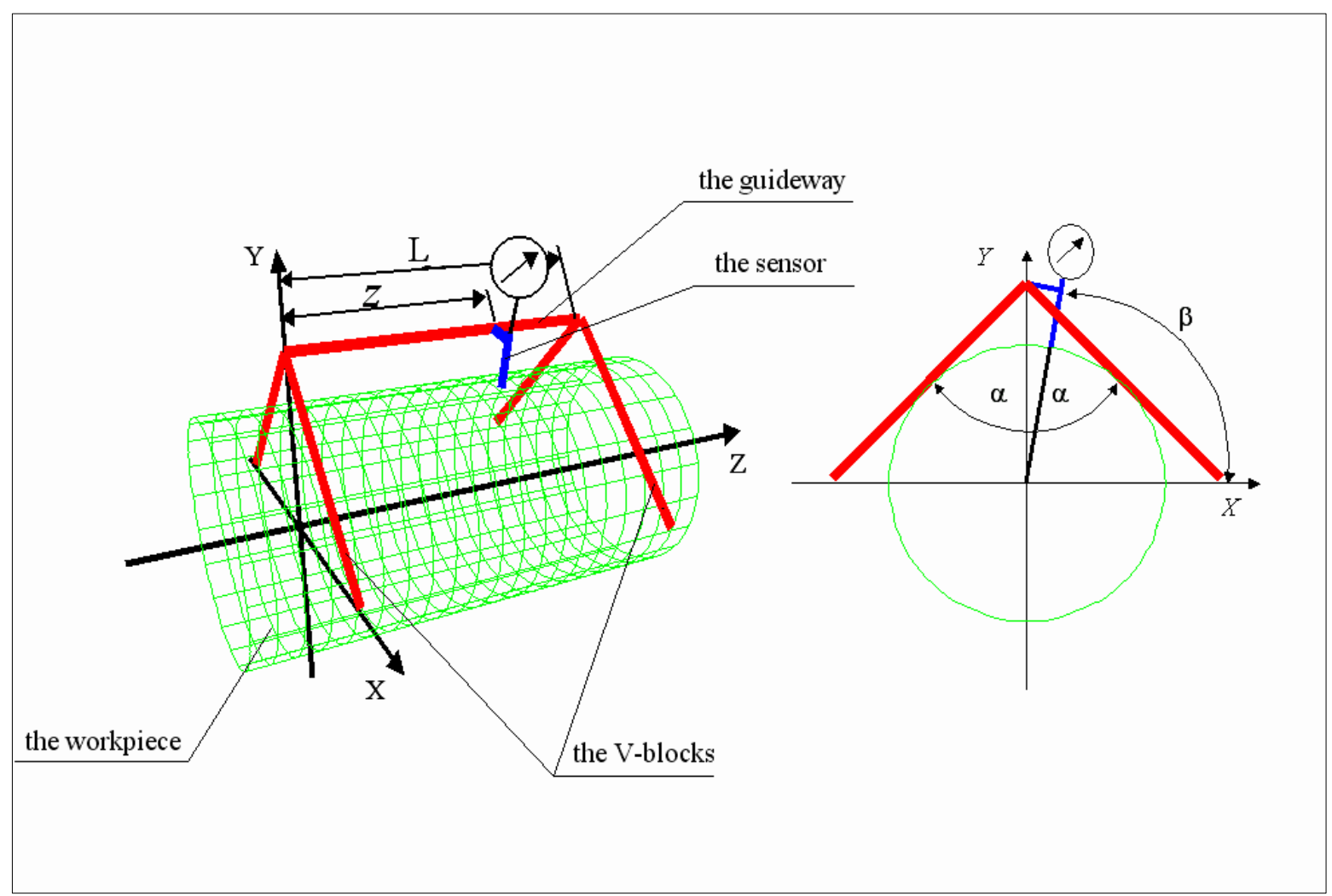

Fig. 2. Principle of measurement of cylidricity profiles with the V-block method, where $\alpha, \beta, \mathrm{L}, \mathrm{z}$ - are method parameters: a) measured workpiece in the XYZ system b) cross-section of a measured workpiece in the plane of the V-block

The proposed concept of cylindricity measurement by means of the V-block method assumes that the measured cylinder is placed on a machine tool (in a centering device). Two interconnected V-blocks adhere to its surface. The connecting element of the V-block functions additionally as a slideway for the measuring sensor. The object's angle of rotation of the cylinder and the sensor's displacement are controlled by the computer. The cylindricity measurement of an object implies appropriate scanning of the cylinder's surface with a measuring sensor, along the 
Stepien, K.; Janecki, D. \& Adamczak, S.: On the Cylindricity Measurement by the ...

suitably designed trajectory, through appropriate steering of the cylinder's angle of rotation and sensor's displacement. Values $\alpha$ and $\beta$ shown in Fig. 2 are the angular parameters of the V-block method for cylindricity measurement. They are responsible for detecting particular harmonic components of the measured cylindricity profile.

The developed concept requires a mathematical transformation of the sensor readings.

\subsection{Mathematical model of the method}

The basis of the relationships presented in this section is the mathematical model of transformation of sensor readings in roundness measurements by the V-block method that was presented in (Adamczak et al. 2010).

The equation of a profile deviation in a cross-section of the cylinder can be best written into the polar co-ordinates system with a centre on the cylinder axis. Let us assume that, if the axis of the nominal cylinder coincides with the axis of the real cylinder, the distance between any point of a profile and the surface of the nominal cylinder is equal to $R(\varphi, z)$, where $\varphi$ and $z$ are co-ordinates of the profile point. If the deviation, $R$, is zero, then the sensor readings do not depend on the co-ordinates $\varphi$ and z. They are proportional to the distance of a given point of a profile from the surface of the nominal cylinder (Adamczak et al. 2011).

Due to the fact that profile deviations are observed at the points of contact of the cylinder's surface and the V-blocks, the real cylinder axis, Z', will deviate slightly from the $\mathrm{Z}$ axis defined by the nominal cylinder axis. We shall denote by $E_{x}(\varphi, z)$ and $E_{y}(\varphi, z)$ the Cartesian co-ordinates of the intersection of the axis of the cylinder turned through the angle $\varphi$ with a plane perpendicular to the $\mathrm{Z}$ axis with the coordinate $\mathrm{z}$ (see Fig. 3). Without the cylinder axis evaluation of cylindricity would not be accurate (Lao et al., 2003).

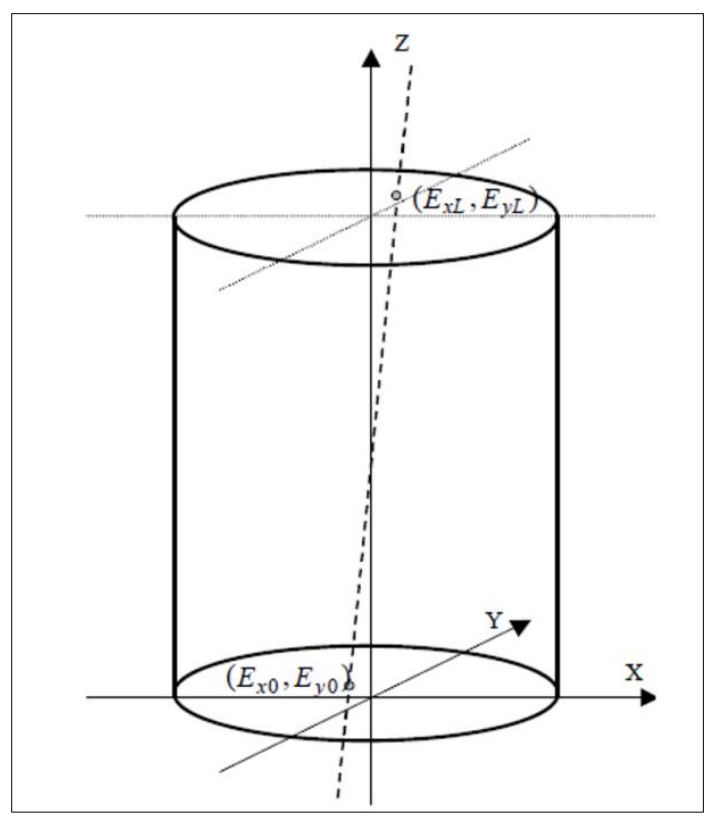

Fig. 3. Cartesian coordinates defining the orientation of the axis of measured cylinder (for given the angle of rotation $\varphi$ ) 
We can determine the values of

$$
E_{x 0}(\varphi)=E_{x}(\varphi, 0), E_{y 0}(\varphi)=E_{y}(\varphi, 0)
$$

and

$$
E_{x L}(\varphi)=E_{x}(\varphi, L), E_{y L}(\varphi)=E_{y}(\varphi, L)
$$

Let us consider the cross-section of the cylinder defined by the plane $z=0$. The points of contact of the measured element and the V-block coincide with the points of contact of the nominal cylinder. So, in these points the distance of the profile from the nominal cylinder is equal to zero. The cylinder rotated through the angle $\varphi$ touches one of the V-blocks at points of a profile with angular co-ordinates $\alpha+\varphi$ and $\pi-\alpha+\varphi$. Thus,

$$
\begin{gathered}
R(\alpha+\varphi, 0)+E_{x 0}(\varphi) \cos \alpha+E_{y 0}(\varphi) \sin \alpha=0, \\
R(\pi-\alpha+\varphi, 0)-E_{x 0}(\varphi) \cos \alpha+E_{y 0}(\varphi) \sin \alpha=0
\end{gathered}
$$

Hence

$$
\begin{gathered}
E_{x 0}(\varphi)=-\frac{R(\alpha+\varphi, 0)-R(\pi-\alpha+\varphi, 0)}{2 \cos \alpha}, \\
E_{y 0}(\varphi)=-\frac{R(\alpha+\varphi, 0)+R(\pi-\alpha+\varphi, 0)}{2 \sin \alpha} .
\end{gathered}
$$

Similarly, analyzing the cylinder cross-section $z=L$, we obtain

$$
\begin{aligned}
& E_{x L}(\varphi)=-\frac{R(\alpha+\varphi, L)-R(\pi-\alpha+\varphi, L)}{2 \cos \alpha}, \\
& E_{y L}(\varphi)=-\frac{R(\alpha+\varphi, L)+R(\pi-\alpha+\varphi, L)}{2 \sin \alpha} .
\end{aligned}
$$

Now the values of the sensor readings $F(\varphi, z)$ for given values of the cylinder's rotation angle $\varphi$ and the displacement of the measuring sensor $\mathrm{z}$ can be determined. They are given by following equation

$$
F(\varphi, z)=R(\varphi+\beta, z)+E_{x}(\varphi, z) \cos \beta+E_{y}(\varphi, z) \sin \beta,
$$

where

$$
\begin{gathered}
E_{x}(\varphi, z)=\frac{E_{x 0}(\varphi)(L-z)+E_{x L}(\varphi) z}{L}, \\
E_{y}(\varphi, z)=\frac{E_{y 0}(\varphi)(L-z)+E_{y L}(\varphi) z}{L} .
\end{gathered}
$$

From the obtained relationships it follows that the value of the deviation can be determined on the basis of the sensor reading, if the co-ordinates of the axis displacement, $E_{x 0}(\varphi), E_{y 0}(\varphi)$ and $E_{x L}(\varphi), E_{y L}(\varphi)$, are known. This, accordingly, requires determining the profiles $R(\varphi, 0)$ and $R(\varphi, L)$. We can achieve this by two additional measurements of roundness profiles in the cross-sections $z=0$ and $z=L$. Then, from Eqs. (5), (6) and (9) we have 
Stepien, K.; Janecki, D. \& Adamczak, S.: On the Cylindricity Measurement by the ...

$$
\begin{aligned}
& F(\varphi, 0)=R(\varphi+\beta, 0)+E_{x 0}(\varphi) \cos \beta+E_{y 0}(\varphi) \sin \beta= \\
& =R(\varphi+\beta, 0)-\frac{1}{2} R(\varphi+\alpha, 0)\left[\frac{\cos \beta}{\cos \alpha}+\frac{\sin \beta}{\sin \alpha}\right]-\frac{1}{2} R(\varphi+\pi-\alpha, 0)\left[-\frac{\cos \beta}{\cos \alpha}+\frac{\sin \beta}{\sin \alpha}\right] .
\end{aligned}
$$

In the above equation, only the profile $R(\varphi, 0)$ is unknown. Thus, the equation should be solved in relation to $R(\varphi, 0)$. The easiest way is to do this in the domain of the coefficients is by expansion of the profile into an exponential Fourier series.

Let $\widehat{F}_{n 0}$ and $\hat{R}_{n 0}$ be the $n$-th components of the expansion of the profiles $F(\varphi, 0)$ and $R(\varphi, 0)$ into an exponential Fourier series $n=-\infty, \ldots,-1,0,1, \ldots, \infty$, i.e.

$$
R(\varphi, 0)=\sum_{n=-\infty}^{\infty} \widehat{R}_{n 0} e^{i n \varphi}, F(\varphi, 0)=\sum_{n=-\infty}^{\infty} \widehat{F}_{n 0} e^{i n \varphi}
$$

Then, we get from (12)

$$
\hat{R}_{n 0}=\frac{\hat{F}_{n 0}}{K_{n}}
$$

where $\hat{K}_{n}$ is the so-called coefficient of detectability defined by

$$
\hat{K}_{n}=e^{i n \beta}-\frac{1}{2} e^{i n \alpha}\left[\frac{\cos \beta}{\cos \alpha}+\frac{\sin \beta}{\sin \alpha}\right]-\frac{1}{2}(-1)^{n} e^{-i n \alpha}\left[-\frac{\cos \beta}{\cos \alpha}+\frac{\sin \beta}{\sin \alpha}\right] .
$$

Similarly, for the profile $z=L$, we get

$$
\hat{R}_{n L}=\frac{\hat{F}_{n L}}{K_{n}} .
$$

Finally, rewriting Eq. (9) in the Fourier coefficients domain, we get the relationship between Fourier expansion coefficients of the sensor readings $\hat{F}_{n}(z)$ and the real signal $\hat{R}_{n}(z)$

$$
\hat{F}_{n}(z)=e^{i n \beta} \hat{R}_{n}(z)-\left(\frac{L-z}{L} \hat{R}_{n 0}+\frac{z}{L} \hat{R}_{n L}\right) \hat{M}_{n},
$$

where

$$
\hat{M}_{n}=\frac{1}{2} e^{i n \alpha}\left[\frac{\cos \beta}{\cos \alpha}+\frac{\sin \beta}{\sin \alpha}\right]+\frac{1}{2}(-1)^{n} e^{-i n \alpha}\left[-\frac{\cos \beta}{\cos \alpha}+\frac{\sin \beta}{\sin \alpha}\right] .
$$

Equations (14)-(18) allow precise calculation of values of Fourier coefficients of the real signal $\hat{R}_{n}(z)$.

Values of real signal $R(\varphi, z)$ can be obtained by inverse Fourier transform of coefficients $\hat{R}_{n}(z)$. It can be easily performed by means of IFFT (Inverse Fast Fourier Transform) algorithm.

\section{Simulations}

On the grounds of the mathematical model of V-block cylindricity measurements, presented in previous section, a set of the computer procedures using the MATLAB package was developed. The developed procedures help prepare qualitative and quantitative evaluation of the cylindricity profiles obtained by $\mathrm{V}$ - 
block methods. In order to verify the correctness of the procedures, computer simulations were conducted.

Computer simulations of the V-block cylindricity measurements were conducted in the following stages:

- the generation of an exemplary cylindricity profile $R(\varphi, z)$,

- the calculation of the measured profile $F(\varphi, z)$ for given method parameters $\alpha, \beta$ and $L$,

- the transformation of profile $F(\varphi, z)$ into the reconstructed profile $R_{p}(\varphi, z)$,

- the determination of the mean cylinder axis for profiles $R(\varphi, z)$ and $R_{p}(\varphi, z)$,

- the comparison of the deviations of profiles $R(\varphi, z)$ and $R_{p}(\varphi, z)$ from the mean axis.

Profile $R(\varphi, z)$ corresponds to the real profile of the workpiece, $F(\varphi, z)$ is the profile obtained directly from the measuring sensor by a V-block method, and $R_{p}(\varphi, z)$ is the profile calculated from profile $F(\varphi, z)$ through its mathematical transformation.

\subsection{Determining the real cylindricity profile}

The real cylindricity profile $R(\varphi, z)$, being the basis for the calculation of profiles $F(\varphi, z)$ and $R_{p}(\varphi, z)$, was designed in order to include all the cylindricity components, described by the standard ISO 12180, i.e. the diameter change in the subsequent cross-sections, the straightness deviation of the cylinder axis and the roundness deviation in individual cross-sections of the cylinder.

The profile is described by the relationship

$$
\begin{aligned}
\mathrm{R}(\varphi, \mathrm{z})=R_{0}+0.001 \cdot \mathrm{R}_{0} & \cdot\left(\sin \left(\frac{\pi \mathrm{z}}{\mathrm{L}}\right)+\frac{10 \mathrm{z}}{\mathrm{L}} \sin \left(\frac{\pi \mathrm{z}}{\mathrm{L}}\right) \sin \varphi+\cos \left(\frac{\pi \mathrm{z}}{\mathrm{L}}\right) \cos \varphi+\sin 2 \varphi+\right. \\
& +5 \cos 3 \varphi+2 \sin 6 \varphi+\cos 10 \varphi+\cos 11 \varphi) .
\end{aligned}
$$

For the purposes of the simulation, the following values of the parameters were assumed:

- the V-block angle $\alpha=60^{\circ}$,

- the angle between the $\mathrm{X}$ axis and the sensor axis $\beta=90^{\circ}$,

- the distance between the prisms $L=200 \mathrm{~mm}$,

- the nominal cylinder radius $R_{0}=20 \mathrm{~mm}$,

- the number of measured cross-sections (including the cross-section of the prisms) $K=11$,

- the number of sampled points per one cross-section $N=1024$,

- the sampling angle $\varphi=\frac{2 \cdot \pi \cdot n}{N}$, where $n=0,1,2, \ldots, N$.

When we have generated profile $R(\varphi, z)$ for given method parameters, i.e. angles $\alpha$, $\beta$ and the distance between the prisms $L$, we can calculate the profile $F(\varphi, z)$, measured by the sensor, from the relationship (9).

As it was mentioned in the previous sections, in order to obtain correct cylindricity parameters by a V-block method, it is necessary to transform the measured profile $F(\varphi, z)$ into the reconstructed profile $R_{p}(\varphi, z)$ using relationships (14) - (18). 
Stepien, K.; Janecki, D. \& Adamczak, S.: On the Cylindricity Measurement by the ...

In order to compare inspected profiles correctly, the orientation of the mean cylinders for the profiles $R(\varphi, z)$ and $R_{p}(\varphi, z)$ has to be calculated (which is tantamount to the calculation of co-ordinates $E_{x 0}, E_{x L}, E_{y 0} E_{y L}$ of the orientation of the mean cylinder axis in the initial and final cross-section of the inspected cylinder), and then both profiles values in relation to the calculated axes have to be determined. In order to determine the orientation of the mean cylinders axes for profiles $R(\varphi, z)$ and $R_{p}(\varphi, z)$, the values of the first harmonic component in all the cross-sections of the cylinders were calculated. Then, co-ordinates $E_{x 0}, E_{x L}, E_{y 0} E_{y L}$ were calculated using relationships (5)-(8). The next step was the calculation of the axis orientation in each cross-section from relationships (10) and (11). The last step was the removing of runout components from the inspected profile, which is the result of the axis shift. After conducting these operations for both profiles, they can be compared.

In order to investigate the conformity of both profiles accurately, the comparison of their subsequent cross-sections was carried out. Figure 4 represents superimposed roundness profiles in the first cross-section of inspected cylinders in Cartesian coordinates. The diagram shown in Fig. 4 confirms that there is a difference between the inspected profiles after considering the orientation of the associated cylinders axes. In order to represent this difference better, the diagram in Fig. 5 represents the bar chart of the amplitudes of subsequent harmonic components (for the range $1 \div 15$ ) of the profiles in the first cross-section of the inspected profiles.

As we can see in Fig. 6, the eleventh harmonic component occurs in the generated profile $R(\varphi, z)$,whereas it does not occur in the processed profile $R_{p}(\varphi, z)$. It is caused by the fact that the selected values of angular parameters $\alpha=60^{\circ}$ and $\beta=90^{\circ}$ do not allow detection the eleventh harmonic component in the generated profile.

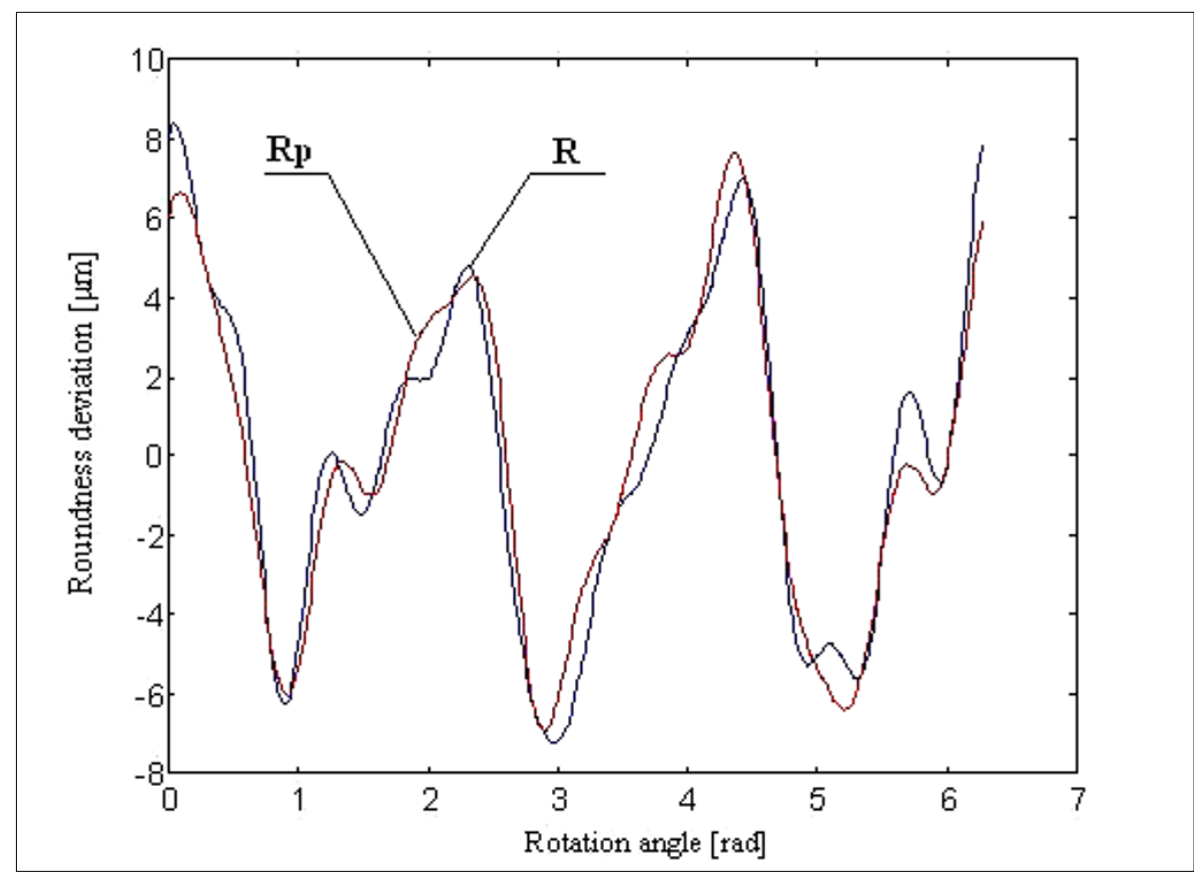

Fig. 4. Superimposed roundness profiles of the first cross-sections of inspected cylinders $R_{p}(\varphi, z)$ and $R(\varphi, z)$ after considering the orientation of the associated cylinders axes 


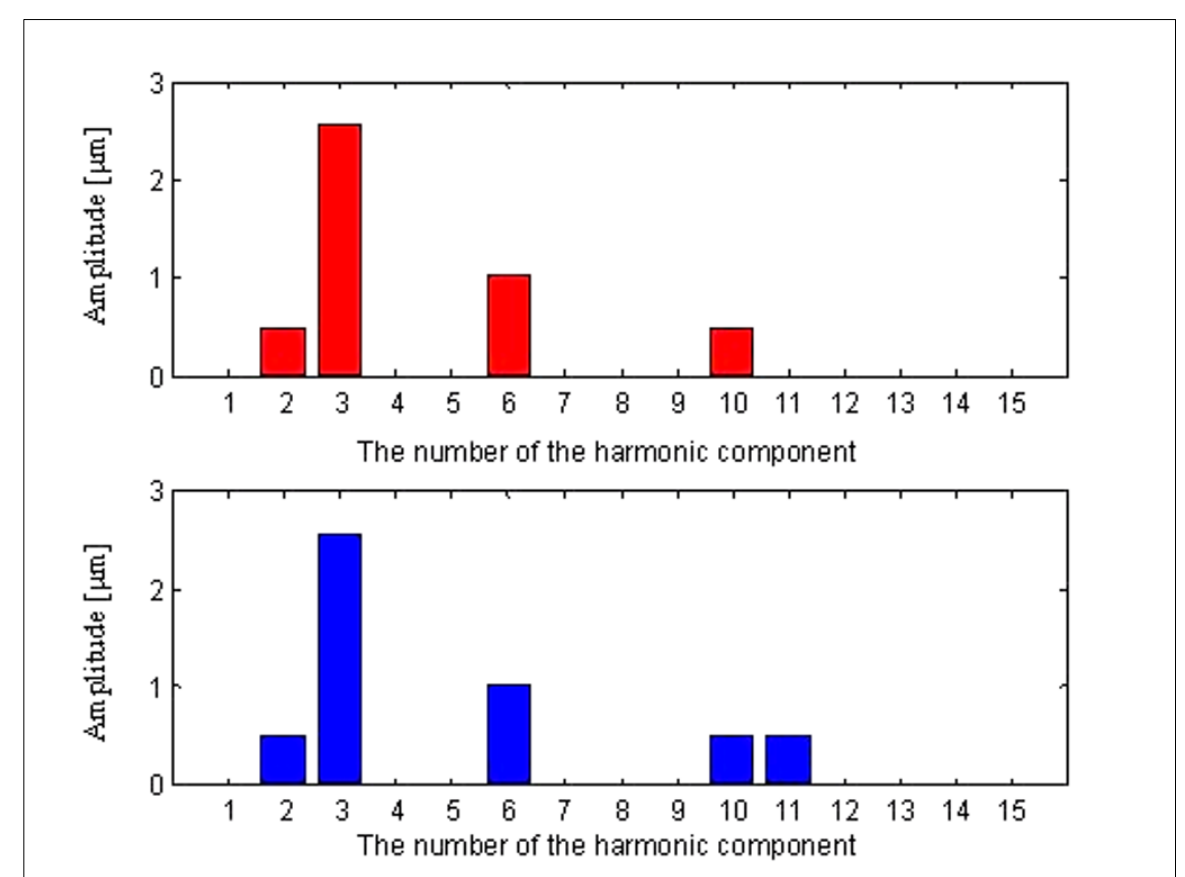

Fig. 5. The bar chart of harmonic components (from 1 to 15) for the profiles; upper chart - the reconstructed profile $R_{p}(\varphi, z)$, lower chart- generated profile

The results of additional computer simulations showed that difference between the values of profiles $R(\varphi, z)$ and $R_{p}(\varphi, z)$ does not change on the entire surface of the inspected cylinder.

Therefore, we can assume that after considering the orientation of the associated cylinder axes, the only significant difference between the profiles $R_{p}(\varphi, z)$ and $R(\varphi, z)$ is the occurrence of the harmonic components which cannot be detected by selected values of the method parameters $\alpha$ and $\beta$. The results of additional computer simulations, show that if generated profile $R(\varphi, z)$ contains only harmonic components detectable by given angular method parameters $\alpha$ and $\beta$, then the difference between inspected profiles is negligible.

\section{Experiment}

\subsection{A measuring instrument for $V$-block cylindricity measurements}

After successful finish of the theoretical research work efforts were taken aiming at practical verification of the developed concept. Therefore it was necessary to design and construct the measuring system allowing cylindricity measurements by the V-block method. Such system has been finally designed and constructed at the Rolling Bearings Factory "Kraśnik" (Poland) in the framework of the research project sponsored by Polish Ministry of Science. The system is shown in Fig. 6. 


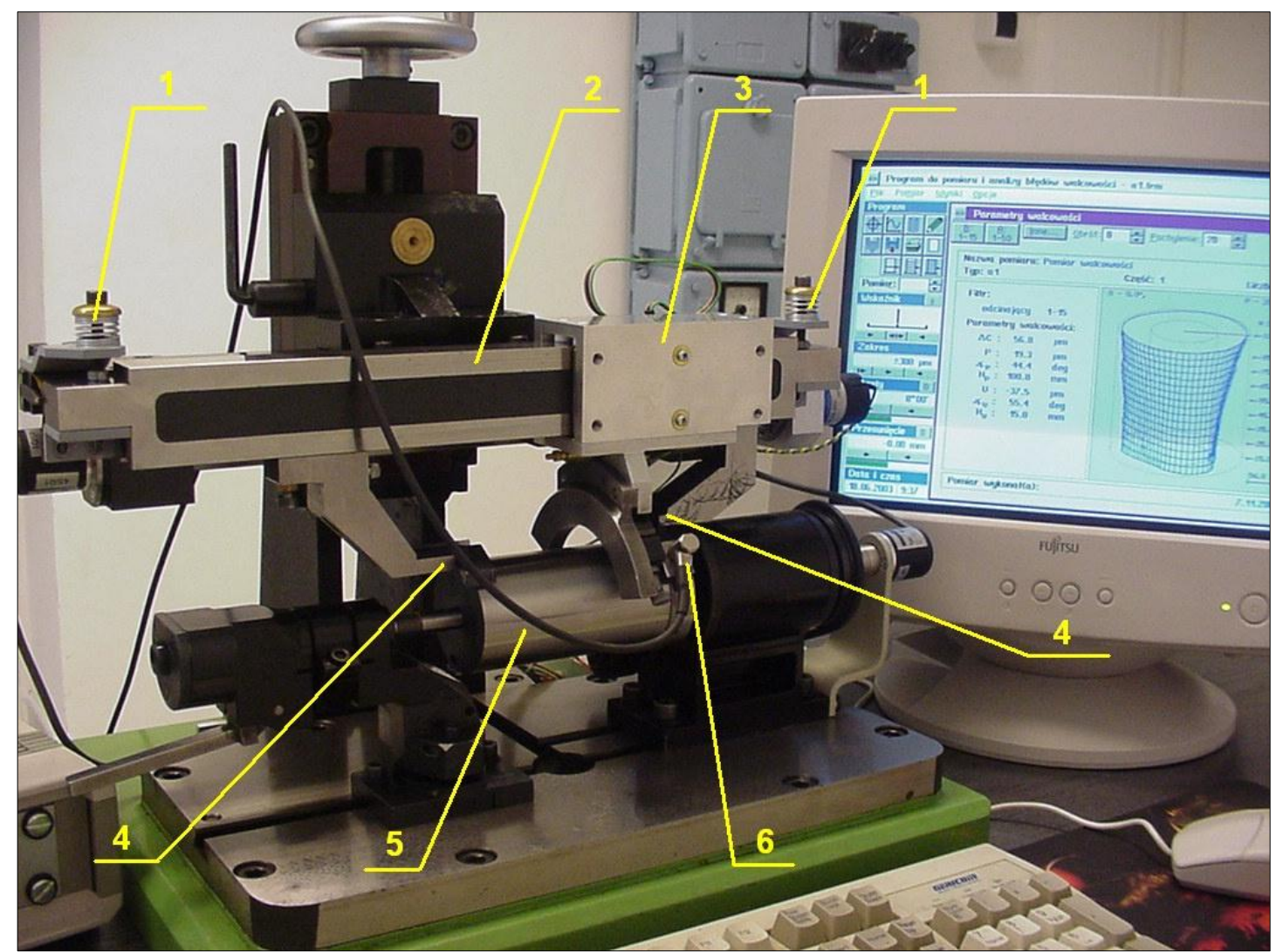

Fig. 6. Model measuring device PSA 6 for the V-block method cylindricity measurements

The testpiece (5) is fixed in the tailstock and in the spindle. The rotation of the workpiece is controlled by the computer. The system of two connected V-blocks (4) lies on the surface of the workpiece. The V-blocks are fixed to the beam (2). The beam (2) is suspended on the system of springs (1). The measuring sensor (6) is fixed to the support (3) that can move along the beam (2). The system was adjusted to the strategy of measurements of subsequent cross-sections. So, roundness profiles in predefined cross-sections of the workpiece are measured. After the measurement the system automatically joins them into cylindrical data.

The device shown in Fig. 6 was used in the experiments aiming at evaluation of the accuracy of the cylindricity measurement by the V-block method.

The experiments involved the comparison of measurement results of the group of cylindrical elements. The results were obtained by the proposed V-block method, using the stand PSA6 and by the radial method, using computer-aided instrument for straightness, roundness and cylindricity measurements Talycenta by Taylor Hobson.

\subsection{Experimental verification of the concept}

Experimental verification of V-block cylindricity measurements involved the comparison of measurement results of the set of eight cylindrical elements, obtained by the V-block method, using the stand PSA6 and by the radial method, using Talycenta. The obtained measurement results were compared using the software, described in the previous subsection. 
When comparing the profiles, the authors focused on observing the occurrence of systematic errors of the V-block method. The comparison was made qualitatively (visually by superimposition compared profiles) and quantitatively - by the calculation of the coincidence coefficient between the profiles and by the comparison of selected cylindricity parameters.

In the experiment the following parameters were analyzed:

- a cylindricity deviation $\mathrm{CYL}_{\mathrm{t}}$,

- a maximum peak over the associated cylinder $\mathrm{CYL}_{\mathrm{p}}$,

- a minimum valley below the associated cylinderCYL $\mathrm{L}_{\mathrm{v}}$,

- a conicity angle $\mathrm{CYL}_{\mathrm{at}}$ and

- a straightness deviation of the cylinder mean line $\mathrm{STR}_{\mathrm{t}}$ ).

Table 1 gives the above mentioned parameters calculated for the measured profiles of the set of seven cylindrical elements.

\begin{tabular}{|c|c|c|c|c|c|c|}
\hline \multirow[t]{2}{*}{ No. } & \multirow{2}{*}{$\begin{array}{c}\text { Measuring } \\
\text { device }\end{array}$} & \multicolumn{5}{|c|}{ Parameters } \\
\hline & & $\begin{array}{l}\mathrm{CYL}_{t} \\
(\mu \mathrm{m})\end{array}$ & $\begin{array}{l}\mathrm{CYI}_{\mathrm{p}} \\
(\mu \mathrm{m})\end{array}$ & $\begin{array}{l}\mathrm{CYI}_{\mathrm{\gamma}} \\
(\mu \mathrm{m})\end{array}$ & $\begin{array}{l}\mathrm{CYL}_{\mathrm{at}} \\
\text { (deg) }\end{array}$ & $\begin{array}{l}\mathrm{STR}_{t} \\
(\mu \mathrm{m})\end{array}$ \\
\hline \multirow{2}{*}{1} & $\overline{\text { PSA } 6}$ & 32,7 & 13,9 & 18,8 & 0,29 & $\approx 0$ \\
\hline & Talycenta & 29,4 & 10,5 & 18,9 & $-0,07$ & $\approx 0$ \\
\hline \multirow[b]{2}{*}{2} & $\overline{\text { PSA } 6}$ & 50,6 & 17,2 & 33,4 & $-0,13$ & $\approx 0$ \\
\hline & Talycenta & 44,6 & 20 & 24,6 & $-0,25$ & $\approx 0$ \\
\hline \multirow{2}{*}{3} & $\overline{\text { PSA } 6}$ & $\overline{41,3}$ & 20 & 21,3 & 0,29 & $\approx 00$ \\
\hline & Talycenta & 37,6 & 14,9 & 22,7 & 0,02 & $\approx 0$ \\
\hline \multirow[b]{2}{*}{4} & $\overline{\text { PSA } 6}$ & 38,7 & 17 & 21,7 & $\overline{00,6}$ & $\approx 0$ \\
\hline & Talycenta & 34,5 & 14,6 & 19,9 & $-0,04$ & $\approx 0$ \\
\hline \multirow[b]{2}{*}{5} & $\overline{\overline{P S A} 6}$ & 42,3 & 16,7 & 25,6 & 0,32 & $\approx 0$ \\
\hline & Talycenta & 37,5 & 12,5 & -25 & $-0,13$ & $\approx 0$ \\
\hline \multirow[b]{2}{*}{6} & $\overline{\text { PSA } 6}$ & 34,4 & 10,3 & 24 & 0,17 & $\approx 0$ \\
\hline & Talycenta & 32,8 & 10,8 & 22 & $-0,12$ & $\approx 0$ \\
\hline \multirow[b]{2}{*}{7} & PSA 6 & 31,4 & 8,2 & 23,2 & 0,1 & $\approx 0$ \\
\hline & Talycenta & 31,4 & 9,1 & 22,3 & $-0,35$ & $\approx 0$ \\
\hline
\end{tabular}

Tab. 1. The results of comparative cylindricity measurements conducted by the Vblock and the radial method

The analysis of the results obtained by the reference and radial methods shows that they are similar. In most cases the difference between values of cylindricity deviation is equal to about a few micrometers. Taking to account that the V-block method is 
Stepien, K.; Janecki, D. \& Adamczak, S.: On the Cylindricity Measurement by the ...

investigated as the method that could be applied under industrial condition, such difference is sufficient.

In Fig. 7 diagrams useful for qualitative comparison of the profiles are shown. The diagrams were generated using the software based on the procedures developed fort he purpose of computer simulations. Each compared pair of profiles was represented in a $3 \mathrm{D}$ diagram at first. If more accurate analysis was required, the diagrams for individual cross-sections were plotted, e.g. polar and Cartesian diagrams of roundness profiles.

a)

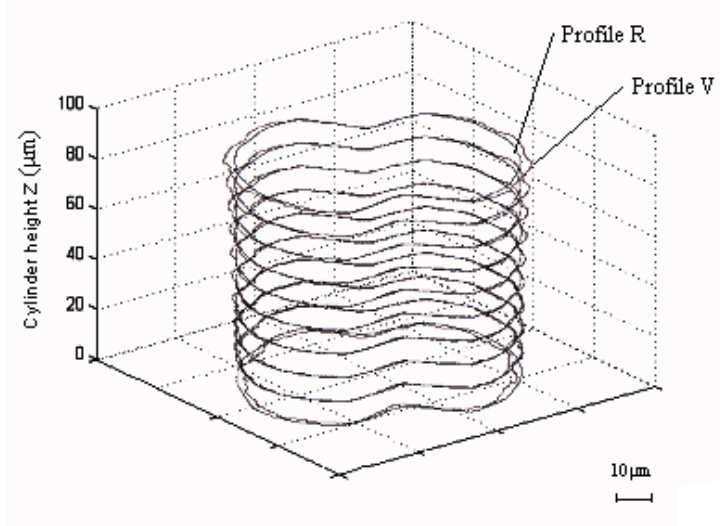

b)

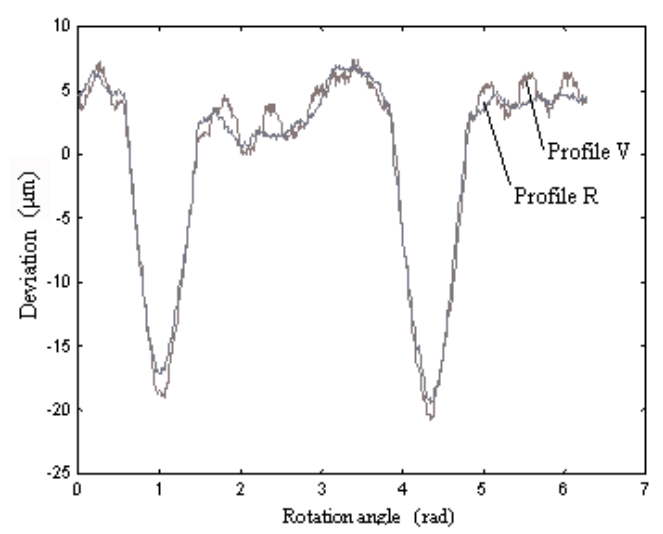

Fig. 7. Comparison of the profiles for the cylinder no. 4, obtained by the V-block method (profile V) and the radial method (profile R): a)a 3D diagram, b) a diagram of roundness profiles in one of the cylinder's cross-sections

\subsection{Statistical research}

In order to establish the accuracy of the V-block method of cylindricity measurement quantitatively a statistical research was carried out.

The statistical verification of the proposed method under industrial conditions was carried out at "Kraśnik" Rolling Bearings Factory. The aim of the test was to determine the method error of V-block measurement of cylindricity profiles for selected sample of elements by means of statistical parameters (taking into account the values of the cylindricity deviation $C Y L t$ ), and to estimate the coefficient of coincidence of the compared profiles defined on the basis of the standardized function of cross correlation.

The relative error of the measurement of the cylindricity deviation $\mathrm{w}_{\mathrm{CYL}}$ was calculated for each element using this relationship:

$$
w_{\mathrm{CYLt}}=\left|\frac{\Delta \mathrm{C}_{\mathrm{o}}-\Delta \mathrm{C}_{\mathrm{p}}}{\Delta \mathrm{C}_{\mathrm{p}}}\right| \cdot 100 \%
$$

where:

$\Delta \mathrm{C}_{\mathrm{o}}-$ cylindricity deviation calculated from the profile obtained with the V-block method, 
$\Delta \mathrm{C}_{\mathrm{p}}-$ cylindricity deviation calculated from the profile obtained with the accurate radial method.

On the basis of relative error the method accuracy (MA) can be calculated from the formula:

where

$$
M A=\bar{w}_{C Y L t} \pm u \cdot s
$$

$M A$ - method accuracy,

$\bar{w}_{C Y L t}$ - mean value of the relative error $w_{C Y L t}$,

$u$ - standardized variable (dependent on reliability level),

$s-$ standard deviation of the relative error $w_{C Y L t}$.

The number of samples was 25 . Thus, twenty five specially machined cylinders and twenty five randomly selected cylinders were used for the laboratory tests and factory verification, respectively. In the calculations reliability level $P=0,95$ was assumed. For this value, $u=1,96$. The results of the calculations are provided in the Table 2 .

\begin{tabular}{|l|l|l|}
\hline \multicolumn{2}{|l|}{ Number of samples $n_{s}$} & 25 \\
\hline \multirow{2}{*}{ Observed value of the relative error } & $w_{C Y L t_{t} \min }$ & -0.142 \\
\cline { 2 - 3 } & $w_{C Y L t_{t} \max }$ & 0.124 \\
\hline Mean value of relative error $w_{C Y L t}$ & 0,020 \\
\hline Standard deviation $s$ of the relative error & 0.084 \\
\hline Method accuracy, MA [\%] & 19.4 \\
\hline $\begin{array}{c}\text { Correlation coefficient } r(\tau) \\
\text { Mean value }\end{array}$ & 0.954 \\
Minimum value & 0.793 \\
Maximum value & 0.994 \\
\hline
\end{tabular}

Tab. 2. Results of statistical testing of the experimental method error for V-block cylindricity measurement under industrial conditions and the values of the correlation coefficients

Results of measurements, given in Table 1 show that the cylindricity profiles obtained with the V-block and accurate radial method are characterized by high correlation. This testifies to their good coincidence, illustrated also in the diagrams showing two compared profiles (see Fig. 7).

Statistical investigation of the accuracy of the method, whose results are given in Table 2, show that the result of the cylindricity deviation measurement taken by means of the proposed method lies within the range $19 \%$ in relation to result obtained by the highly accurate radial method (for assumed probability level $\mathrm{P}=0,95$ ). 
Stepien, K.; Janecki, D. \& Adamczak, S.: On the Cylindricity Measurement by the ...

\section{Specific problems about the V-block method of cylindricity measurements}

\subsection{Problems relating to the measurement concept}

The first problem relating to the measurement concept is that some harmonic components cannot be detected by the measuring system. This shortcoming can be compensated by such selection of the method parameters (angles $\alpha$ and $\beta$ in Fig.2) that would permit detection these harmonic components that are most important for the user. Guidelines for selection desired method parameters can be found, for example, in (Sonozaki \& Fujiwara, 1989). Another solution of this problem is such design of the measuring system that allows adjustment of the angles $\alpha$ and $\beta$. Unfortunately, the system shown in Fig. 2 does not allow adjustment of the method parameters. The second problem concerning the practical application of the method is the fact that the mathematical transformation of the measured signal into a processed one is necessary to obtain reliable measurement results. As far as the authors know there is not any commonly available commercial software that for this purpose. Therefore authors developed their own software for such transformation. The software was based on the procedures written in Matlab environment that had been developed on the fundamental of equations given in the section 2. Thus, the measuring system shown in Fig. 6 was used only for data acquisition. Then the measurement data were saved as a text file and transferred to the software operating in the Matlab environment. The software allows measurement data processing, an evaluation of the profile by values of cylindricity deviations and plotting different types of diagrams.

\subsection{Problems relating to the design of the measurement system}

Another practical difficulties that occurred were problems relating to the design of the measuring system. The first one concerned the shape of the contact surfaces of the V-blocks. At the beginning of experiments this shape was sharp in order to assure area of contact of the V-blocks and measured workpiece to be as small as possible. However, it turned out that such design of V-blocks results in damaging of surface of workpieces made of soft steels. Therefore, it was necessary to modify the shape of the contact surfaces of the V-blocks. In final experiments their shape was spherical.

The second factor that had negative influence on measurement results was too stiff suspension of the system: V-blocks- the beam (the guideway) - the sensor. At the beginning of the experiment the beam was suspended by the set of two flat springs. However, it turned out that such suspension is too stiff and was the reason of incorrect sensor readings. Therefore, the design of the system has been modified and stiff flat springs have been replaced by softer coil springs (see elements no. 1 in Fig. $6)$.

\subsection{Errors of measuring system components}

Important problems from the point of view of practical application of V-block methods are errors of components of measuring system. Such errors sometimes influence very significantly on measurement results. For example, during experiments it turned out that profiles measured by the V-block method are 
characterized by conical error. However, measurements of these profiles by the standard, highly accurate radial method showed that this conicity error is only virtual - the real workpiece surface is not conical. Therefore research on the source of this conicity error was done. The result of the research was that virtual conicity of measured profile may appear due to:

- unequal angles of V-blocks,

- the slope of the guideway in relation to the surface of measured workpiece (for example, caused by unequal fixing the $\mathrm{V}$-blocks to the guideway).

In order to investigate the first issue, a computer simulation was made. It showed that even small difference of V-blocks angles influences very significantly on measurement result (Janecki \& Stępień, 2008). For example, difference of the Vblocks angles equal to $0,02^{\circ}$ causes appearance of virtual conicity deviation equal to $11,7 \mu \mathrm{m}$ (simulation was made for the nominal cylinder defined by following values: the radius: $20 \mathrm{~mm}$ and the height $100 \mathrm{~mm}$ ).

Taking to account results of the research the angles of the V-blocks were measured by coordinate measuring machine. It was established that:

- the angle of the left V-block $\alpha_{0}=59^{\circ} 51^{\prime} 27^{\prime \prime}$,

- the angle of the right $\mathrm{V}$ - block.: $\alpha_{\mathrm{L}}=59^{\circ} 51^{\prime} 58^{\prime \prime}$.

Measured values of the V-blocks angles were used to calculate appropriate correction coefficients that were put into the software.

Besides the difference of the V-blocks angles and the guideway slope, influence of other factors on measurement results was also investigated. For example, following problems were investigated: straightness deviation of the guideway, offnominal orientation of the measuring sensor and calibration error of the sensor. It turned out that in the case of the measuring system shown in Fig. 8 these factors hardly influence measurements results.

Important part of the experimental verification of the concept of was visual comparison of profiles obtained by the V-block method and by the accurate radial method regarded as a reference. The visual comparison showed that there were quite large fluctuations of the profiles obtained by the V-block method, which was not observed in the profiles obtained by the radial method (see Fig. 7b)). The source of these fluctuations is not clear. In authors' opinion they may be the result of vibrations of the screw thread mechanism of the measuring sensor drive. However, this problem should be investigated in the nearest future.

\section{Conclusion}

Taking into consideration the requirements of modern technological processes, presented method of the V-block cylindricity measurements seems to be very interesting. Practical advantages of the computer-aided V-block method were shown in the case of roundness measurements. Research work on the application of the Vblock method for roundness measurements has resulted in developing of measurement systems allowing in-situ roundness measurements. Such systems work in industrial plants and universities, such as, for example, Alstom (England) and ABB 
Stepien, K.; Janecki, D. \& Adamczak, S.: On the Cylindricity Measurement by the ...

Brno (Czech Republic). In authors' opinion it is possible to design and construct similar system that could be used also for cylindricity measurements.

In order to make it possible to apply the V-block method to measurements of cylindricity it was necessary to develop a mathematical model of such measurement. Very important part of the model are equations allowing transformation of the measured profile into the reconstructed one. Computer simulations showed that developed model is correct, however, some harmonic components of the profile cannot be detected by the measuring system. Except for this shortcoming, the model is not the source of any other systematic errors.

On the fundamentals of the developed concept a special-purpose measuring system was designed and constructed. The system was used in the experimental verification of the method. Results of measurements, given in Table 1 show that the cylindricity profiles obtained with the V-block and accurate radial method are characterized by high correlation. This testifies to their good coincidence, illustrated also in the diagrams showing two compared profiles (see Fig. 7). Statistical investigation of the accuracy of the method, whose results are given in Table 2, show that the result of the cylindricity deviation measurement taken by means of the proposed method lies within the range $19 \%$ in relation to result obtained by the highly accurate radial method (for assumed probability level $\mathrm{P}=0,95$ ). Assuming that the calculated experimental method error is a sufficient measure of accuracy, and that the accuracy of an instrument to be used for measurement of the geometrical surface structure during the product quality control ranges $10 \%-25 \%$, it is clear that the proposed concept of reference measurement of cylindricity profiles is characterized by sufficient accuracy. The test results indicate that the V-block method is applicable to cylindricity measurement, and that the specially constructed measuring system can be used under industrial conditions. Note, that workpieces, whose cylindricity deviation was equal to about $30 \div 50 \mu \mathrm{m}$ were measured. In authors' opinion, taking to account the current state-of the-art on the V-block cylindricity measurement, this method should not be applied in measurements of workpieces, whose deviation is smaller.

There were a lot of practical problems that occured in the experimental part of the research work.

The first one was necessity to develop suitable software allowing transformation of the sensor readings into the reconstructed profile. Without such transformation measurement results would be completely wrong.

The second problem was that some harmonic components could not be detected by the measuring system. This shortcoming, characteristic feature of all V-block methods, can be compensated, for example, by adjustment of the angular method parameters (angles $\alpha$ and $\beta$ from Fig. 2). Unfortunately, the measuring system used in the experiments does not allow adjustment of the method parameters.

Another practical difficulties concerned the mass and stiffness of the measuring system. It turned out in the case of measurement of workpieces made of soft steels, contact surfaces of the V-blocks can damage the surface of the workpiece. It may be caused, for example, by too large mass of the V-blocks system, sharp shape of the contact surfaces of the V-blocks and too stiff suspension of the measuring system. 
Therefore, engineers, who would design V-block systems in future, should take into consideration following questions:

- shape of measuring surfaces of V-blocks should not be sharp,

- the mass of the V-blocks system should be rather small,

- the suspension of the system should not be too stiff.

Other factors that influenced measurement results were errors of the components of the measuring system. For, example, when analyzing measurement results it turned out that profiles measured by the V-block method were characterized by a conicity deviation. However, the same profiles measured by the radial method used as a reference, were not conical. Therefore, authors assumed that the deviation of conicity is only virtual and it is caused by geometrical deviations of the components of the $\mathrm{V}$ block measuring system. Investigation on this problem showed that virtual conicity deviation can be caused, for example, by the difference of the V-blocks angles. Therefore, very important issue is measurement of real values of the V-blocks and putting proper correction coefficients into the software.

Another good solution allowing calculation of the correction coefficients could be measurement of the element, whose profile is known (for example, if it was measured earlier by the measurement instrument of high accuracy). Difference between the measured and the reference profile would allow identification of such errors as slope and straightness deviation of the guideway or calibration error of the measuring sensor and other ones. Of course, such error as the straightness deviation of the guideway could be also identified by direct measurement with an interferometer or other measuring instrument for straightness measurement.

Visual comparison of profiles measured by the V-block and the radial method showed that the profile obtained by the V-block is characterized by larger fluctuations that the one measured by the radial method. The reason of this phenomenon is not quite clear. In authors' opinion it is probably caused by the vibrations of the screw thread drive of the sensor. However, this problem should be investigated in near future.

Despite the problems with the implementation, the V-block method of cylindricity measurements is very promising. In authors' opinion most of the problems can be solved, which would make it possible to design and construct the Vblock system for cylindricity measurement. Such system would allow accurate in-situ measurement of form deviations of large cylinders that are used, for example, in paper or electric power industry. In authors' opinion design, building up and testing of such system should be the next stage of the research work on V-block cylindricity measurements.

\section{References}

Adamczak S., Janecki D., Stępień K. (2010). Qualitative and quantitative evaluation of the accuracy of the V-block method of cylindricity measurements. Precision Engineering, Vol. 34, No.3, 619-626, ISSN: 0141-6359 
Stepien, K.; Janecki, D. \& Adamczak, S.: On the Cylindricity Measurement by the ...

Adamczak S., Janecki D., Stępień K. (2011). Cylindricity measurement by the Vblock method - Theoretical and practical problems. Measurement, Vol. 44, No. 1, 164-173, ISSN: 0263-2241

Beranek, L.; Volf, L. \& Mikes, P. (2011). Coordinate Metrology Education Using Virtual CMM, Annals of DAAAM for 2011 \& Proceedings of the 22nd International DAAAM Symposium, 23-26th November 2011, Vienna, Austria, Volume 22, No. 1, ISSN 1726-9679, ISBN 978-3-901509-83-4, Katalinic, B. (Ed.), pp. 1323-1324, Published by DAAAM International Vienna, Vienna

Gao W, Kiyono S. (1997). On-machine roundness measurement of cylindrical workpieces by the combined three-point method. Measurement, Vol. 21, No. 4, 147-156, ISSN: 0263-2241

Janecki D., Stępien K. (2008). A study of the effects of the sensor guideway slope on the results of the reference measurement of cylindricity deviations. Pomiary Automatyka Kontrola, Vol. 5, 258-262, ISSN 0032-4140

Katalinic, B. (2010). Engineers for Knowledge Based Society, Annals of DAAAM for 2010 \& Proceedings of the 21st International DAAAM Symposium, 2023rd October 2010, Zadar, Croatia, ISSN 1726-9679, ISBN 978-3-901509-735, Katalinic, B. (Ed.), pp. 0001-0002, Published by DAAAM International Vienna, Vienna

Lao. Y.-Z. et al. (2003). Accurate cylindricity evaluation with axis-estimation preprocessing. Precision Engineering, Vol. 27, No. 4, 429-437, ISSN: 01416359

Muralikrishnan B., Venkatachalam S., Raja J., Malburg A. (2005). A note on the three-point method for roundness measurement. Precision Engineering, Vol. 29, No. 2, 257-260, ISSN: 0141-6359

Okuyama E., Goho K., Mitsui K. (2003). New analytical method for V-block threepoint method. Precision Engineering, Vol. 27, No. 3, 234-244, ISSN: 01416359

Runje, B. \& Medic, S. (2011). Measurement Uncertainty in Process of Line Scales Calibrating, Annals of DAAAM for 2011 \& Proceedings of the 22nd International DAAAM Symposium, 23-26th November 2011, Vienna, Austria, Volume 22, No. 1, ISSN 1726-9679, ISBN 978-3-901509-83-4, Katalinic, B. (Ed.), pp. 0093-0094, Published by DAAAM International Vienna, Vienna

Sonozaki S., Fujiwara H. (1989). Simultaneous measurement of cylindrical parts profile and rotating accuracy using multi-three-point- method. Bulletin of Japan Society of Precision Engineering, Vol. 23, No. 4, 286-291, ISSN: 01416359

Zhao W.Q. et al. (2006). SSEST: A new approach to higher accuracy cylindricity measuring instrument. International Journal of Machine Tools and Manufacture, Vol. 46, No. 14, 1869-1878, ISSN 0890-6955 